\title{
Effect of Annealing and Time of Crystallization on Structural and Optical Properties of PVDF Thin Film Using Acetone as Solvent
}

\author{
Imtiaz Noor Bhatti ${ }^{1}$, M. Banerjee ${ }^{2}$ and Ilyas Noor Bhatti ${ }^{3}$ \\ ${ }^{I}$ (Nanoscience and Nanotechnology lab. School Of Physics, D.A.V.V. Indore, India) \\ 2 (Nanoscience and Nanotechnology lab. School Of Physics, D.A.V.V. Indore, India) \\ ${ }^{3}$ (Laser Building, School Of Physics, D.A.V.V. Indore, India)
}

\begin{abstract}
In this report pure poly(vinylidene fluoride) (PVDF) films were prepared by casting method using acetone solvent. The crystallization of both $\alpha$ and $\beta$ phase from acetone solvent by varying the time of crystallization has been described. This paper also describes the enhancement of $\beta$ phase at different annealing condition. $\beta$ phase dominant thin films were obtained when as cast thin films were annealed at $90{ }^{\circ} \mathrm{C}$ for 5 hours. The PVDF films with dominant $\alpha$-phase were obtained, when time of crystallization is extend. From ( $X$ ray diffraction) XRD and Fourier Transform Infrared Spectrum (FTIR) it is confirmed that the PVDF thin films, cast from acetone solution and annealed at $90{ }^{\circ} \mathrm{C}$ for 5 hours, have maximum percentage of $\beta$-phase. Presence of the crystalline $\alpha$ and $\beta$ phases in each sample was confirmed by X-ray Diffraction (XRD) and Fourier transform infrared spectroscopy (FTIR). We found that of PVDF when crystallized from its acetone solutions led to the formation of $\beta$ phase. UV-visible optical absorption analysis revealed a change in the optical gap and shift in absorption edge with annealing temperature.
\end{abstract}

Keywords: Annealing, casting, polymorphism, polar, PVDF

\section{INTRODUCTION}

The introduction Poly(vinylidene fluoride), $\mathrm{PVDF}\left(\mathrm{CH}_{2}-\mathrm{CF}_{2}\right)$ is a semi- crystalline polymer which has, in recent times, attracted the attention of scientists due to its pyro and piezoelectric properties. Another interesting property of PVDF is its polymorphism. PVDF exists in five crystalline phases- $\alpha$ phase which is nonpolar, while $\beta, \delta, \gamma$ and $\varepsilon$ phases which are polar. Although PVDF has a simple chemical structure, it is well established that it can exhibit five different polymorphs depending on its processing conditions. These crystal forms involve three different chain conformations, namely: (1) all-trans (TTTT) planar zigzag for $\beta$-phase, (2) TGTG' for $\alpha$ and $\gamma$ phases, and (3) $\mathrm{T}_{3} \mathrm{GT}_{3} \mathrm{G}^{\prime}$ for $\delta$ and $\varepsilon$ phases [1]. When PVDF chains are packed into crystal lattices, their dipoles are either additive, which leads to a net dipole as in $\beta, \gamma$ and $\delta$ phases, or cancelled among themselves, resulting in no net dipole as in $\alpha$ and $\varepsilon$ phases [1]. Among the three polar phases, the $\beta$-phase has the largest spontaneous polarization (p) per unit cell and thus exhibits the highest piezo-, pyro and ferroelectric activities, which endues PVDF with great potentials for various device applications[2]. However, the phases of a particular PVDF film depend on the method of preparation and post preparation treatments. Various methods have been used to prepare PVDF films, like melt casting, spin coating, solution casting, chemical vapor deposition etc. Post preparation treatments include mechanical stretching, stretching along with heating, electric poling, annealing etc.[3][4][5]. Many researchers intend to enhance the $\beta$ phase of PVDF and it is frequently investigated worldwide.

Researchers had adopted different methods reported in literatures. The effect of filler like $\mathrm{BiCl}_{3} \mathrm{FeCl}_{3}$ $\mathrm{CoCl}_{2}$ and $\mathrm{MgCl}_{2}$ on the content of the $\alpha$ and $\beta$ phases and the structural and physical properties of PVDF films is reported [6],[7]. These techniques normally induced undesired structural deformation giving rise to changes of the physical properties in the worse direction. Effect of annealing on phase transition in poly(vinylidene fluoride) films prepared using DMSO solvent. [8] When deposited from solutions PVDF commonly crystallizes in $\alpha$ and $\gamma$ phase. The $\beta$ phase has been found in solvent-cast thin films when a highly polar but toxic solvent, hexamethyl phosphoramide, was used [5]. Solution casting method has become popular recently and has been established as a simple and versatile method for fabricate polymer thin films. The solvents used frequently by various researchers are highly polar solvents like DMF and DMSO. There is no report on the systematic study of PVDF films prepared using acetone as a solvent. This motivated us to investigate the possibility of preparing PVDF films by solution casting method using a less polar solvent like acetone.

This work was undertaken to investigate the crystalline phase variation in PVDF thin films under various conditions using acetone solvent. In the present study we have prepared PVDF films by solution casting method using acetone as solvent under two set of conditions. The preparation conditions have been varied to obtain two kinds of films. Then in post preparation treatment films were subjected to thermal annealing for five hour. An as-cast PVDF film is kept for reference study and three films annealed at different temperature from 
each set are studied using XRD, FTIR and UV-Vis. spectroscopy. Finally effect of annealing and time of crystallization on structural and optical properties PVDF films have been studied and reported in this report.

\section{Methods ANd Procedure}

\section{Materials}

PVDF powder used in the study obtained from Alfa Aeser Chemical Company and Acetone obtained from RFCL Limited (RENKEN). The double distilled water was prepared in laboratory.

\section{Experimental}

\subsection{Sample Preparation}

Crystallization from solution method is used for sample preparation. Films of PVDF were prepared by casting method. The solvent used was the pure acetone to dissolve the PVDF powder. PVDF was dissolved in the acetone at PVDF concentration, namely polymer/solvent weight ratio of $15 \mathrm{wt} . \%$. Solution was heated at 60 ${ }^{\circ} \mathrm{C}$ for 30 minutes with continuous stirring to make it clear. The solution of dissolved polymer were cast on glass and dried at room temperature. Imposing condition on crystallization time PVDF films were casted under two sets.

2.1.1 Under Set-1 the films were cast on glass substrate. These films were dried rapidly at room temperature in 30 minutes.

2.1.2 Under set-2 the time of crystallization was increased. This was achieved by reducing the evaporation rate of solvent. The solution content kept in beakers covered with aluminum foil and kept undisturbed to drying. It took 25 days to completely dry and we obtained transparent PVDF self standing films (foil).

The films so produced were kept at room temp. for two weeks to ensure the complete removal of solvent traces . The films so produced were annealed at three different temperatures viz. 70,90 and $110^{\circ} \mathrm{C}$. Then four films viz. as cast, annealed at 70,90 and $110^{\circ} \mathrm{C}$ of each set were characterized by XRD, FTIR and UV-Vis spectroscopy. For XRD films on glass substrate were used where as self standing films were used for FTIR and UV-VIS spectroscopy.

\subsection{Characterization techniques}

The XRD patterns were recorded using D8 Discover diffractometer from Bruker. The XRD patterns were recorded $2 \theta$ ranging $10^{\circ}$ to $50^{\circ}$ with step scanning was performed at $0.05^{\circ}$ intervals and CuKa radiation $(\lambda=$ $1.54 \AA$ ) was used as the X-ray source. The FTIR spectra in the wave number range $400-4000 \mathrm{~cm}^{-1}$ were recorded in transmissions mode using Vertex 70 FTIR spectrometer from Bruker. UV-Vis Spectra were recorded using Shimadzu 2450 spectrophotometer. The absorption spectra were measured in wavelength range $200-1100 \mathrm{~nm}$.

\subsection{X-Ray diffraction}

\section{RESULT AND DisCUSSIONS}

The XRD scans of PVDF films set-1 and set-2 are shown in Fig. 1.1 and 2.1 respectively. The general feature of the observed spectra indicates the presence of a semicrystalline structure. The XRD pattern of rapid dried as cast PVDF film showed amorphous nature of sample with signature of peak around $2 \theta=20.20^{\circ}$. This is because of rapid drying which froze the mono-mers of PVDF in random that found no time to crystallize. Whereas XRD pattern of slowly crystallize as cast film is semicrystalline with characteristics peaks at $2 \theta=$ $18.46^{\circ}, 20.45^{\circ}, 26.70^{\circ}$ and $39.20^{\circ}$. The peaks at $18.46^{\circ}$ and $39.20^{\circ}$ assigned to $\alpha$-phase whereas peak at $20.45^{\circ}$ was assigned as $\beta$-phase [3],[5],[6]. When the rapid dried and slowly crystallized films thermally annealed at 70 , 90 and $110^{\circ} \mathrm{C}$ for 5 hour peaks appear and assigned to different phases of PVDF which are tabulated in Table 1. The XRD patterns of rapid dried films conforms that the presence of $\beta$-phase around $2 \theta=20.23^{\circ}$ the peak become prominent with increase in annealing temperature up to $90^{\circ} \mathrm{C}$. The $\beta$-phase is dominant in which was film rapid dried and annealed at $90^{\circ} \mathrm{C}$ for 5 hour. The films slowly crystallized result in dominant $\alpha$ phase because monomers of PVDF had enough time to settle to stable crystalline structure. The XRD patterns of these films have prominent peak at $2 \theta=18.60^{\circ}$ and $39.30^{\circ}$ assigned to $\alpha$-phase. There is no effect of annealing on $\alpha-$ phase while peaks assigned to $\beta$-phase at $20.40^{\circ}$ got intense a bit.

\subsection{Fourier Transform Infra-red Spectroscopy}

Figure 1.2 and 2.2 shows the FTIR spectrums of the set- 1 and set-2 pure PVDF films. According to literature, the a-phase shows the characteristic absorption bands at $975 \mathrm{~cm}^{-1}$ (CH2 twisting), $796 \mathrm{~cm}^{-1}$ (CH2 rocking), 763 and $616 \mathrm{~cm}^{-1}$ (CF2 bending and skeletal bending), $532 \mathrm{~cm}^{-1}$ (CF2 bending) and $489 \mathrm{~cm}^{-1}$ (CF2 bending) while $\beta$-polymorph has peaks at $510\left(\mathrm{CF}_{2}\right.$ bending $)$ and $840 \mathrm{~cm}^{-1}\left(\mathrm{CH}_{2}\right.$ rocking mode)[8][9][10]. The spectra contain clear absorption peaks characterizing both $\alpha$ and $\beta$-phase. The peaks at 511, 840 and 886 are figure prints of $\beta$ phaseand peaks at $616 \mathrm{~cm}^{-1} 763 \mathrm{~cm}^{-1}$ and $975 \mathrm{~cm}^{-1}$ are assigned to $\alpha$-phase. The spectra contain 
clear peaks characterizing $\beta$ phase occurs most notably at $511,839 \mathrm{~cm}^{-1}, 886 \mathrm{~cm}^{-1}$ and $1173 \mathrm{~cm}^{-1}$ and $\alpha$ phase at $430,616,796$ and $975 \mathrm{~cm}^{-1}$. In order to compare the content of each crystalline phase, a characteristic IR frequency bands for each phase is required. In this study infrared bands at $839 \mathrm{~cm}^{-1}$ and $616 \mathrm{~cm}^{-1}$ were used in the analysis as the characteristic bands for $\beta$ and $\alpha$ phase respectively.

The FTIR spectra of films conforms the $\beta$ and $\alpha$ phases exist. It is found that with increase in annealing temperature absorption intensity at $616,763 \mathrm{~cm}^{-1}$ sharply decreases whereas the absorption intensity increases at510 and $840 \mathrm{~cm}^{-1}$. It advocates the increase in $\beta$ phase of PVDF is observed in PVDF films of set- 1 . In the FTIR spectral analysis of set-2 films it become clear that shifting of band positions observed but $\alpha$-phase peak height remained unaltered, however minute absorption band at $510 \mathrm{~cm}^{-1}$ signifies presence of $\beta$-phase. The above discussion of FTIR conform the x-ray diffraction results.

\subsection{Optical absorption}

The UV/VIS optical absorption was measured for the present PVDF system. The UV-visible absorption spectroscopy technique is used for the investigation of optical properties of the PVDF polymer samples in the wavelength range 190-800 nm. The UV-visible spectra of set-1 and set-2 PVDF films are shown in Fig.1.2 and 2.2 respectively. The absorption of light energy by polymeric materials in the ultra violet and visible region involves the promotion of electrons in $\sigma, \pi$ and n-orbitals from the ground state to higher energy state [11]. The optical absorption spectrum of the as-cast film in Fig. 1.2(a) shows that the absorption decreases sharply from $190 \mathrm{~nm}$ to $220 \mathrm{~nm}$ and then decreases gradually up to $320 \mathrm{~nm}$. In between $320 \mathrm{~nm}$ and $360 \mathrm{~nm}$, two distinct characteristics peaks are observed which may be correlated with electronic transition occurring in fluorocarbon (CF). After $360 \mathrm{~nm}$ the optical absorption decreases linearly at higher wavelength. The fundamental absorption shows a sudden rise in absorption, known as absorption edge, which can be used to determine the optical band gap $(\mathrm{Eg}=\mathrm{h} \mathrm{c} / \mathrm{v})$. Absorption is expressed in terms of a coefficient $\alpha(v)$ (absorption coefficient), which is defined as the relative rate of decrease in light intensity. The absorption coefficient $\alpha(v)+$ was calculated from the absorbance $(\mathrm{P})$. Where $\alpha(v)=2.303 \mathrm{P} / \mathrm{d}$ and $\mathrm{d}$ is the sample thickness.

Band gap analysis

In order to study the effect of structural change due to annealing time of crystallization on optical behaviour of PVDF films, optical band gap (Eg) for PVDF films has been determined using Tauc's relation equation-(1) [8].

$(\alpha h v)=\mathrm{A}\left[\mathrm{h} v-\mathrm{E}_{\mathrm{g}}\right]^{\mathrm{n}}$

where $\mathrm{h} v$ is the photon energy, $\mathrm{h}$ is Planck's constant, $\alpha$ is the absorption Coefficient, $\mathrm{E}_{\mathrm{g}}$ is the optical energy gap, $\mathrm{A}$ is the constant, for direct transitions $\mathrm{n}=1 / 2$. We plot a graph between $(\alpha \mathrm{h} v)^{2}$ versus $\mathrm{h} v$, the linear part of each tauc curve in Fig. 1.4 indicates the presence of an optical gap Eg. The extrapolation of the straight line to $(\alpha h v)^{2}=0$ axis gives $E_{g}$. Figure 1.4 and 2.4 shows the plots of $(\alpha h v)^{2}$ versus $h v$ for the set- 1 and set -2 PVDF films respectively. The fundamental absorption edge is one of the most important features of the absorption spectrum of crystalline and amorphous materials. Is found absorption edge shifted to longer wavelength with increase in annealing temperature. The values of absorption edge and energy band gap $\mathrm{E}_{\mathrm{g}}$ obtained for both set of films is tabulated in table 2. It is found that the band gap of PVDF films decrease with increase in annealing temperature and absorption edge shifted to longer wavelength. Further rapid dried films has lower band gap as compared to films crystallized slowly under set-2 as shown in fig 3 . The decrease in band gap is a result of increase in crystallite size in PVDF films. Since band gap is inversely related to crystallite size.

\section{FIGURES AND TABLESS}
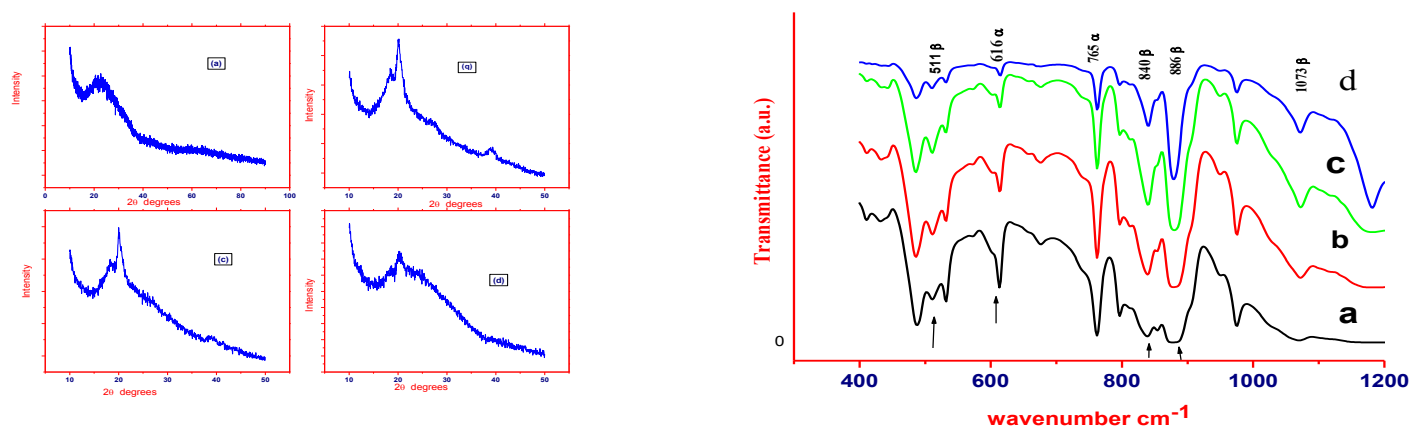

Fig.1.2 XRD pattern of PVDF set-1 films (a) as cast film, (b), (c) and (d) are films annealed at 70, 90 and $110^{\circ} \mathrm{C}$ respectively Optical spectra for set 1 PVDF films

Fig.1.2 FTIR spectra of PVDF set-1 films (a) as cast film, (b), (c) and (d) are films annealed at 70, $90 \quad$ and $\quad 110 \quad{ }^{\circ} \mathrm{C} \quad$ respectively. 

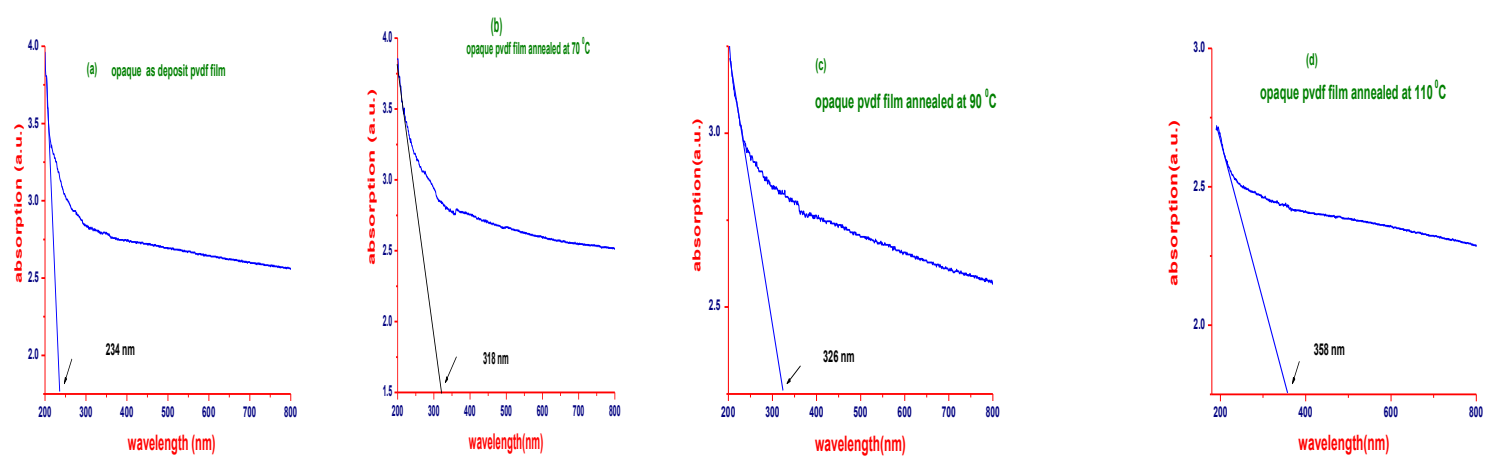

Figure 1.3 Absorption plot of PVDF set-1 films (a) as grown film, (b), (c),and (d) for samples annealed at 70,

Taus's Plot:
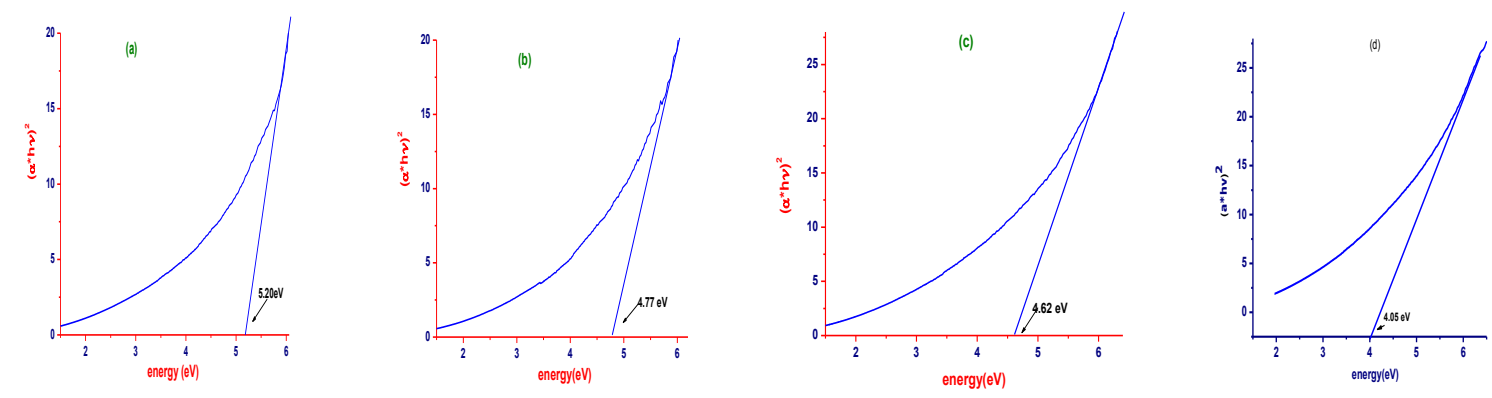

Figure1.4 Square of alpha*energy Vs energy plots of set-1 films (a) As cast film. (b), (c) and (d) are films annealed at 70,90 and $110^{\circ} \mathrm{C}$.
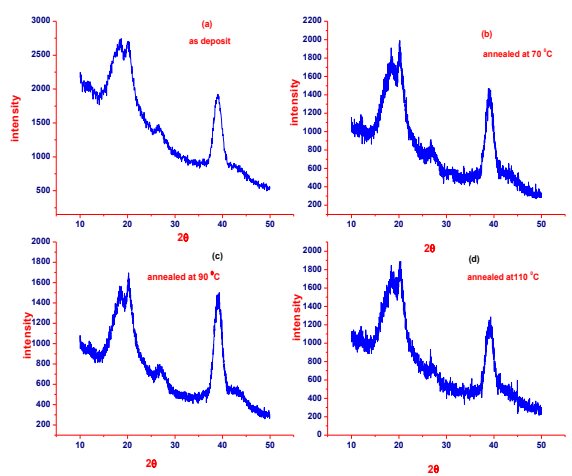

Figure 2.1 XRD patterns for set -2 films, (a) as cast film, (b) films annealed at $70{ }^{\circ} \mathrm{C}$, (c) films annealed at $90^{\circ} \mathrm{C}$ and (d) films annealed at $110^{\circ} \mathrm{C}$.

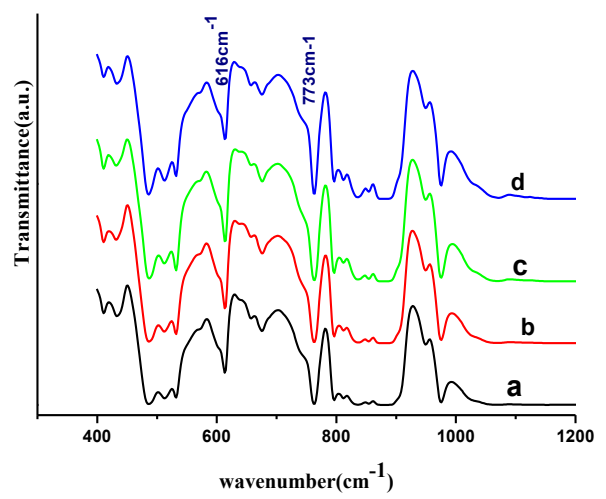

Figure 2.2 FTIR patterns for set -2 films, (a) as cast film, (b) films annealed at $70{ }^{\circ} \mathrm{C}$, (c) films annealed at $90{ }^{\circ} \mathrm{C}$ and (d) films annealed at $110^{\circ}$
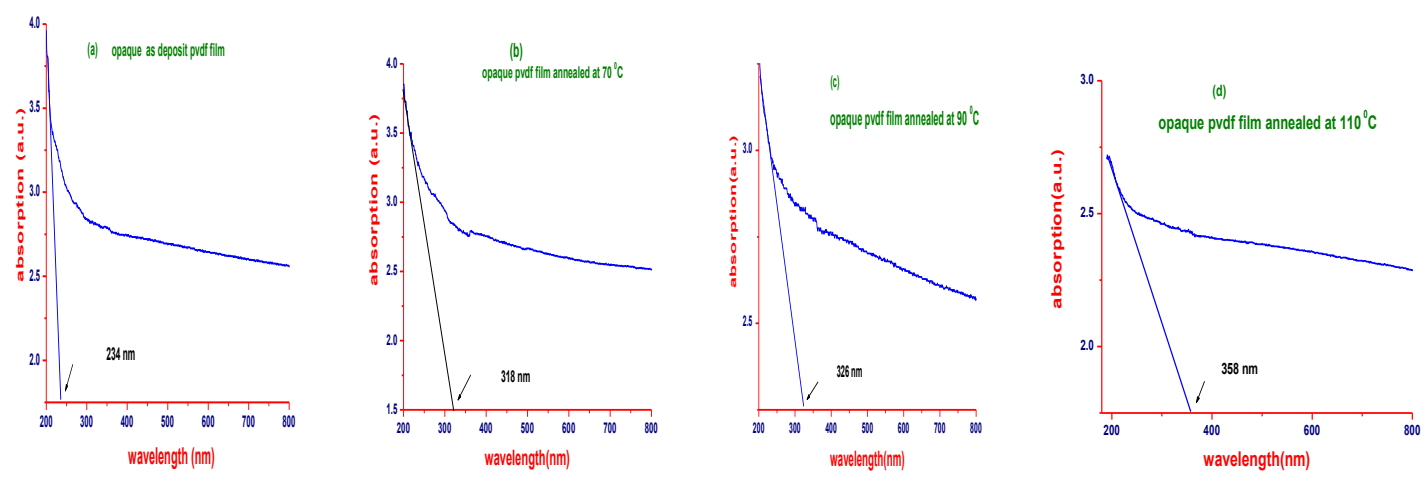
Figure 2.3 Absorption plot of PVDF set - 2 film (a) As cast film. (b), (c) and (d) are films annealed at 70, 90 Tauc's plot:
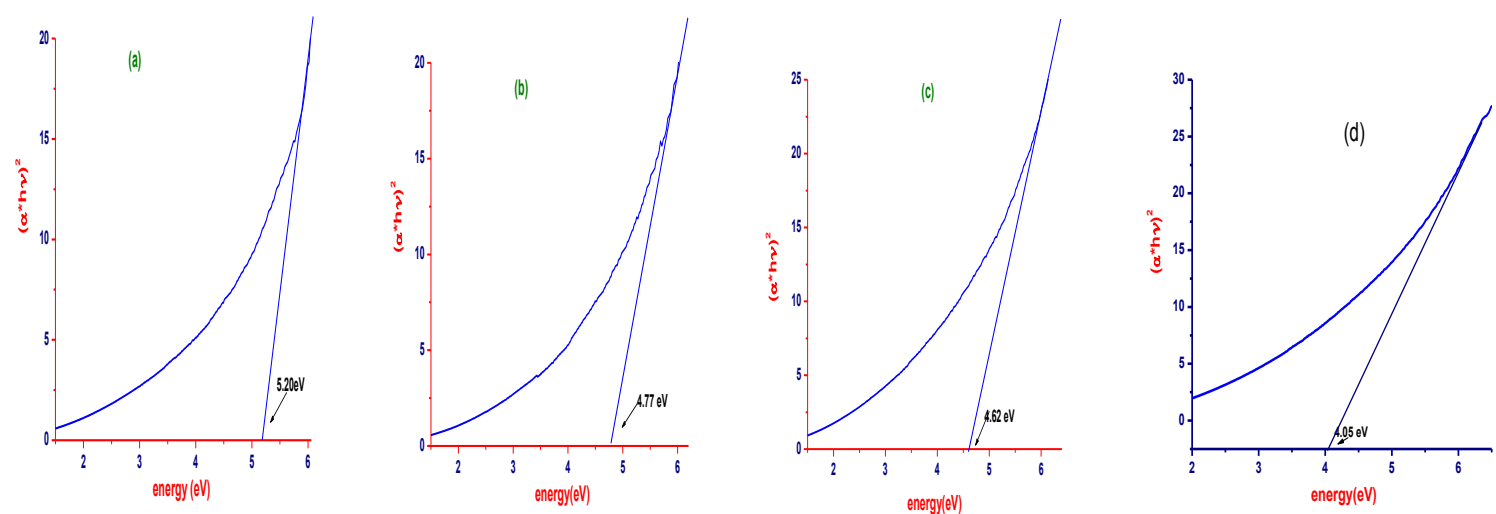

Figure 2.4 Square of alpha*energy Vs energy for PVDF set - 2 films (a) As cast film. (b), (c) and (d) are films annealed at 70,90 and $110{ }^{\circ} \mathrm{C}$.

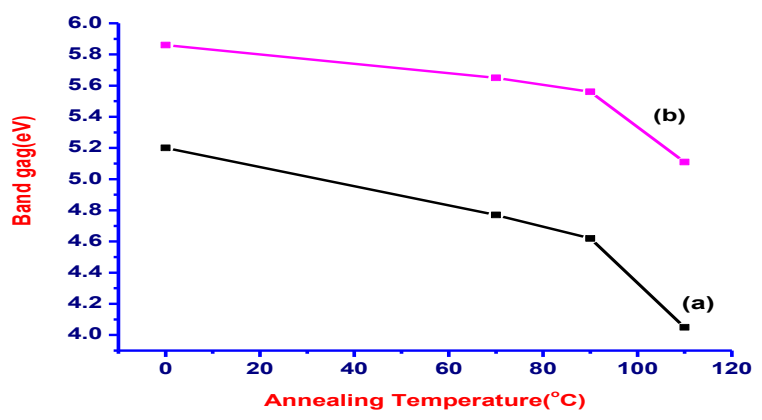

Figure 3.1 Band gap against annealing temperature (a) and (b) for samples prepared under set -1 and set -2 conditions respectively

TABLES

\begin{tabular}{lcccc}
\hline & \multicolumn{2}{c}{ Set -1} & \multicolumn{2}{l}{ Set -2} \\
\hline Sample & $2 \theta$ & Assignment & $2 \theta$ & \multicolumn{1}{c}{ Assignment } \\
\hline As grown film & 20.6 & $\beta$ phase & 18.46 & $\alpha$ phase \\
& 22.6 & $\alpha$ phase & 20.45 & $\beta$ phase \\
& 42.7 & $\alpha$ phase & 26.80 & $\alpha$ phase \\
\hline Annealed at $70^{\circ} \mathrm{C}$ & & 39.20 & $\alpha$ phase \\
& 18.4 & $\alpha$ phase & 18.46 & $\beta$ phase \\
& 20.2 & $\beta$ phase & 20.42 & $\gamma$ phase \\
& 26.7 & $\gamma$ phase & 26.80 & $\alpha$ phase \\
\hline Annealed at $90^{\circ} \mathrm{C}$ & 39.6 & $\alpha$ phase & 39.30 & $\alpha$ phase \\
& 18.2 & $\alpha$ phase & 18.59 & $\gamma$ phase \\
& 20.1 & $\beta$ phase & 20.40 & $\alpha$ phase \\
\hline Annealed at $110^{\circ} \mathrm{C}$ & 38.4 & $\alpha$ phase & 26.80 & $\alpha$ phase \\
& 18.6 & $\alpha$ phase & 39.30 & $\beta$ phase \\
& 20.1 & $\beta$ phase & 18.30 & $\alpha$ phase \\
\hline
\end{tabular}

Table1 Peaks feature in XRD patterns of samples under study.

\begin{tabular}{|l|l|c|l|c|c|}
\hline $\begin{array}{l}\text { S. } \\
\text { no. }\end{array}$ & Samples & \multicolumn{2}{|l|}{ Set - 1 PVDF thin films } & Set - 2 PVDF films & Band gap (eV) \\
\cline { 3 - 6 } & & Absorption Edge $\lambda(\mathrm{nm})$ & Band gap $(\mathrm{eV})$ & Absorption Edge $\lambda(\mathrm{nm})$ & 5.86 \\
\hline 1. & As deposit & 227 & 5.20 & 238.8 & 5.65 \\
\hline 2. & Annealed at $70^{\circ} \mathrm{C}$. & 320 & 4.77 & 233.3 & 5.56 \\
\hline 3. & Annealed at $90^{\circ} \mathrm{C}$. & 326 & 4.62 & 240.6 & 5.11 \\
\hline 4. & Annealed at $110^{\circ} \mathrm{C}$ & 358 & 4.05 & 296 & \\
\hline
\end{tabular}

Band gap and absorption edge of PVDF samples: 
Table-2 Experimental values of band gap and absorption edge for under study samples.

\section{CONCLUSION}

In this report it is observed that the same solvent can yield all the three phases of PVDF. By changing the time of crystallization different phases of PVDF can be obtained using less polar acetone solvent. The time of crystallization and annealing conditions decide the presence of different phases and conversion of one phase to other phase of PVDF. The $\beta$-phase which is important for ferroelectric application is obtained using less polar solvent and by suitable annealing. Maximum $\beta$-phase exists in the rapidly dried PVDF films when are annealed at $90^{\circ} \mathrm{C}$ for $5 \mathrm{~h}$. for. The enhanced $\beta$-phase at different annealing condition has been confirmed from XRD and FTIR of PVDF film. Thus dominant $\beta$-phase films can be prepared under rapid drying of solution and annealing at $90^{\circ} \mathrm{C}$ for 5 hour. Films produced by slow crystallization are rich in stable $\alpha$-phase and remain unaltered after annealing. UV-VIS study shows presence of absorption edge in each case. The position of absorption edge shifts to longer wavelength with annealing temperature. Energy band gap is calculated using tauc plot and it is found that annealing effect band gap. The decrease in energy band gap for each film is observed. Thus films with wholly $\beta$-phase can be produced by less polar solvents.

\section{REFERENCES}

[1] Lovinger, A. J. Ferroelectric polymers. Science 220,1115 1121 (1983).

[2] Nalwa, H. S. Ferroelectric Polymers (Dekker, 1995).

[3] S. Eleshmawi, "Spectroscopic, Thermal, and Electrical Investigations of PVDF Films Filled with BiCl3". Journal of Applied Polymer Science, Vol. 102, 2125-2131 (2006)

[4] A. Tawansi, "FeCl3-CoCl2 mixed fillers effects on the structural, electrical and magnetic properties of PVDF films." Journal of Magnetism and Magnetic Materials 262 (2003) 203-211

[5] S. Satapathy "Effect of annealing on phase transition in poly(vinylidene fluoride) films prepared using polar solvent." Bull. Mater. Sci., Vol. 34, No. 4, July 2011, pp. 727-733.

[6] A. Tawansi "Structural and electrical properties of MgCl2-filled PVDF Films." Polymer Testing 18 (1999) 569-579.

[7] Vipin Kumar, Sachin Kr. Sharma, T.P. Sharma and V. Singh, "Band gap determination in thick from repentance measurements." Elsevir Optical Materials 12 (1999) 115-119.

[8] Ali Akbar Yousefi, "Influence of Polymer Blending on Crystalline Structure of Polyvinylidene Fluoride". Iranian Polymer Journal, 20 (2), 2011, 109-121

[9] B. Jaleh • P. Fakhri • M. Noroozi • N. Muensit, "Influence of Copper Nanoparticles Concentration on the Properties of Poly(vinylidene fluoride)/Cu Nanoparticles Nanocomposite Films" J Inorg Organomet PolymDOI 10.1007/s10904-012-9660-5

[10] A. Rawat, H. K. Mahavri, S Chauhan, A.Tanwar \& P. J. Singh, "optical band gap of polyvinylpyrrolidone/polyacrilamide blend thin films”. Indian Journal of Pure \& Applied Physics, Vol. 50, February 2012, pp. 100-104 